\title{
Interface bonding performance of CuW alloy coating on Cu substrate
}

\author{
Jiliang Zhang ${ }^{1,}$ a, Fei Wang ${ }^{1}$, Jie Yan $^{1}$, Yonghua Gu ${ }^{1}$, Kaiyong Jiang ${ }^{1}$ \\ ${ }^{1}$ Key Laboratory of Digital Measurement Technology, Huaqiao University, Xiamen, 361020, China \\ aemail: Mikeswell@hqu.edu.cn
}

Keywords: CuW Coatings; Cu substrate; Microwave sintering; Interface; Wedged load test

\begin{abstract}
In this paper, CuW alloy is successfully coated on copper substrate by microwave sintering. A wedged load test and scanning electronic microscopy (SEM) are employed to investigate the interface bonding performance. The results show that the $\mathrm{CuW}$ coating bonds firmly to the copper substrate without any gap or crack. The interface bonding strength increases with the temperature increase, but doesn't show an obvious regularity changing with holding time increase.
\end{abstract}

\section{Introduction}

$\mathrm{Cu}-\mathrm{W}$ is an important alloy which combines the features of tungsten and copper. CuW coating on copper substrate will enhance some properties of copper, such as higher melting point, welding resistance and arc erosion resistance, while this composite will retain the advantages of copper, such as high electrical conductivity, high thermal conductivity and well processing workability. $\mathrm{CuW} / \mathrm{Cu}$ composite is widely used in high voltage switch, relays, EDM electrodes and other electronic applications.[1-3] Because tungsten and copper are immiscible and the melting point of tungsten is very high, conventional preparation of $\mathrm{CuW}$ alloy is to sinter the mixed powders of copper and tungsten. But traditional powder metallurgy methods have encountered some difficulties, such as longer sintering time, higher temperature and higher energy consumption.

Microwave sintering is an innovative process of powder metallurgy technology which has been used in preparing materials from 1980. Since Gavin Whittaker et al introduced this technology into metal powder sintering field, many alloys have been manufactured for many applications. [4, 5] Compared with conventional sintering technology, it has the advantages of lower temperature, shorter duration, higher efficiency and better properties of product in density, hardness and so on.

In this paper, we present a method to manufacture $\mathrm{CuW}$ coatings on copper substrate by microwave sintering. The interface between $\mathrm{CuW}$ coating and copper substrate is experimentally investigated. The influence of temperature and holding time on the interface bonding strength is also studied and discussed.

\section{Experiment}

Commercially available pure copper powders (purity $>99.9 \%$, particle size $<25 \mu \mathrm{m}$, provided by Shanghai Jingchun Ltd., China) and pure tungsten powders (purity $>99.5 \%$, particle size $<2 \mu \mathrm{m}$, provided by Xiamen Jinlu Ltd., China) were employed to prepare the coatings. The powders were mixed with different composition in a planetary ball mill (XQM-2L, Nanjing Daran Ltd. China) with $300 \mathrm{r} / \mathrm{min}$ for $8 \mathrm{~h} .80^{\circ} \mathrm{C}$ vacuum drying for $4 \mathrm{~h}$ is subsequent.

The pure copper substrates were cut in pieces with a size of $12 \mathrm{~mm} * 10 \mathrm{~mm} * 3 \mathrm{~mm}$ by wire electrical discharge machine (WEDM). To ensure the good bonding performance between coatings and substrate, a series of post-process on copper substrate including degreasing, acid pickling, ultrasonic cleaning and vacuum drying was necessary. After that, the mixed CuW powders were pressed on the $\mathrm{Cu}$ substrate by a tablet machine (769YP-24B, Tianjing Keqi, China) with a pressure of 20MPa and a holding time of 2 min.

The green samples were covered with $\mathrm{SiC}$ pieces and sintered in a $2.45 \mathrm{GHz} 3 \mathrm{~kW}$ high vacuum microwave sintering furnace (HAMiLab-HV3, Changsha Longtai co., Ltd., China). The pressure was less than $10^{-3} \mathrm{~Pa}$ and the heating rate was $15^{\circ} \mathrm{C} / \mathrm{min}$. The sintering temperature were set to $850^{\circ} \mathrm{C}, 880^{\circ} \mathrm{C}, 920^{\circ} \mathrm{C}$ and the holding time were set to $5 \mathrm{~min}, 10 \mathrm{~min}$ and $15 \mathrm{~min}$, respectively. 
Detail process was illustrated in other paper. [6]

Optical microscopy (VHX-1000, Keyence, Japan) and scanning electronic microscopy (S-3500N, Hitachi, Japan) were employed to observe the interface morphology. The specimen used to observe was prepared under condition of $880^{\circ} \mathrm{C}, 10 \mathrm{~min}$ holding time.

Because the coating thickness is about $300-400 \mu \mathrm{m}$, conventional adhesion tests such as scratch test and shear test are unsuitable for this research. Adhesive strength test result also showed that the interface bonding strength is higher than the epoxy adhesive strength (20MPa). A wedged load strength test was employed to measure the interface bonding strength. [7] In additional, for avoiding slip and damage of the CuW coating, a copper block was introduced to enhance the damage resistance of $\mathrm{CuW}$ coatings. The block combined to the $\mathrm{CuW}$ coating surface with an epoxy binder. The test principle and process is schematically illustrated in Fig 1. The maximum shear strength of interface can be deduced and described as follow:

$$
\sigma_{\max }=C \frac{p_{\max }}{2 \omega h_{2}} \cot \frac{\alpha}{2}-1.5 p_{\max } \frac{\left(\frac{L_{0}}{2}-h_{2} \tan \frac{\alpha}{2}\right)}{\omega h_{1}^{2}}
$$

In this equation: $\sigma$ is the shear strength of interface which we are concerning; $p$ is the load; $\alpha$ is the sharp angle of the stainless steel indenter; $\omega$ is the width; $L_{0}$ is the total length of complex; $h_{2}$ is the depth of initial slot; $h_{1}$ is height of specimens without count in $h_{2}$; C is a coefficient of correction which is 1.5 in this paper.

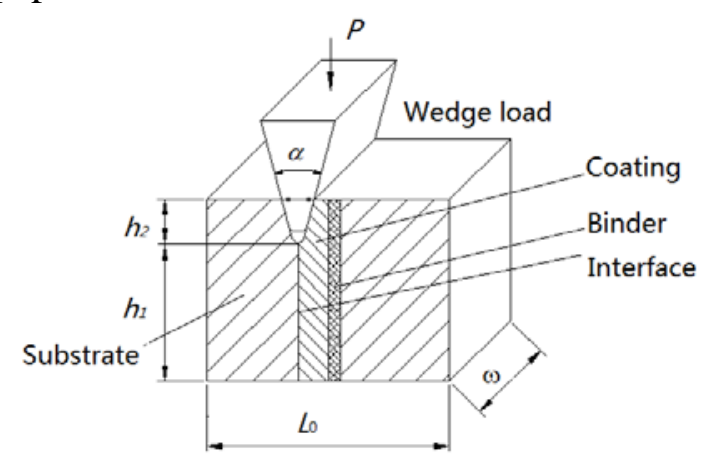

Fig.1 Schematic of the wedged load test

\section{Results and discussion}

Fig. 2 shows the cross section morphology of the $\mathrm{CuW}$ coating on $\mathrm{Cu}$ substrate $\left(880^{\circ} \mathrm{C}, 10 \mathrm{~min}\right)$. It can be seen that the white tungsten particles distribute in the coating dispersedly. The thickness of the CuW coating in this experiment is about $300-400 \mu \mathrm{m}$, according to the dosage of the CuW mixed powders. Not any gap or crack in the interface can be observed in this figure. This result indicates that the interface bonding performance is very well and the CuW coating can bond firmly to the copper substrate by the process we present.

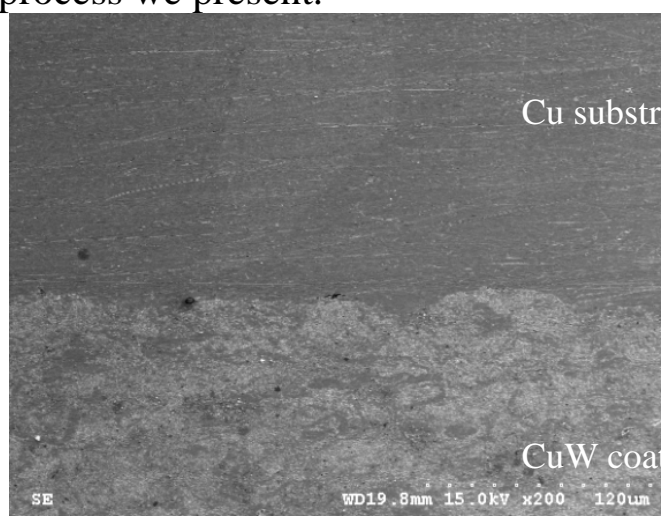

Fig.2 The cross section morphology of CuW coating on Cu substrate

After wedged load test, fracture morphologies of the specimen are shown in Fig.3. The CuW coating is cut off integrally from the copper substrate along the interface strictly. It can be seen 
some copper material transfer from the substrate to the $\mathrm{CuW}$ coating. The transfer pit can reach up to a depth of $150 \mu \mathrm{m}$ and area of $20 \mathrm{~mm}^{2}$. This result indicates that the bonding strength between $\mathrm{CuW}$ coating and $\mathrm{Cu}$ substrate is higher than the copper cohesion in some place, particularly the defects of copper substrate. Due to the good interface bonding performance, the defects (gaps or cracks) in copper substrate near the interface are going to grow up, even peel off in the loading procedure. Consequently, the copper exfoliation bonds to the $\mathrm{CuW}$ coating interface firmly and transfers in the separation of $\mathrm{CuW}$ coating and $\mathrm{Cu}$ substrate.

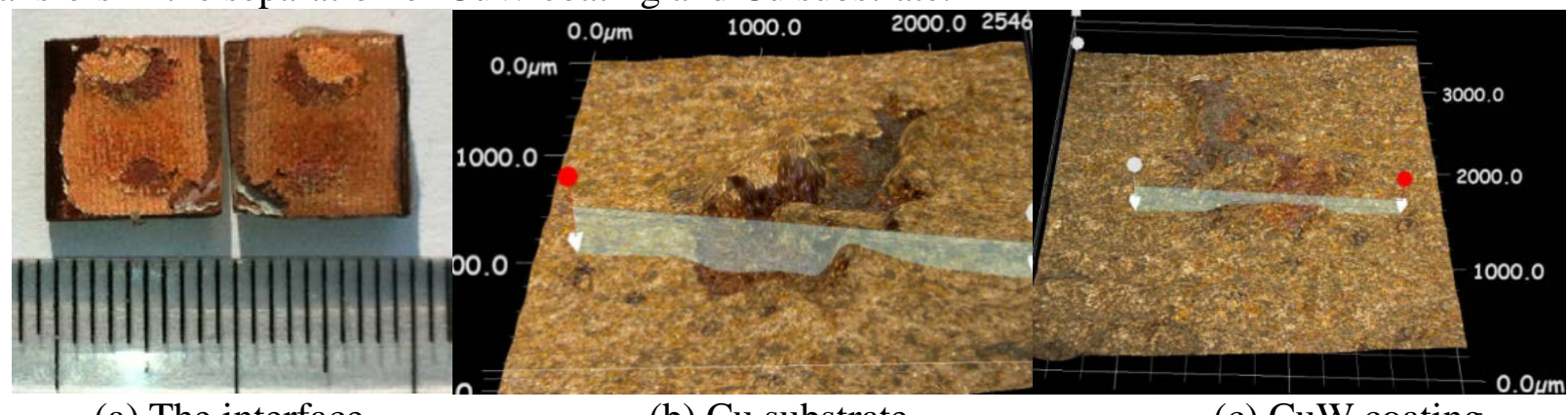

(a) The interface

(b) Cu substrate

(c) $\mathrm{CuW}$ coating

Fig.3 The fracture morphology of $\mathrm{Cu}$ substrate and $\mathrm{CuW}$ coating after wedged load test

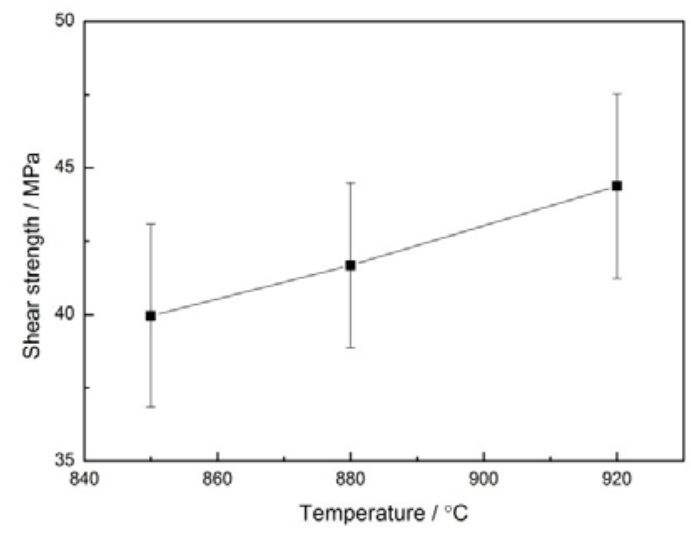

(a) Different temperature

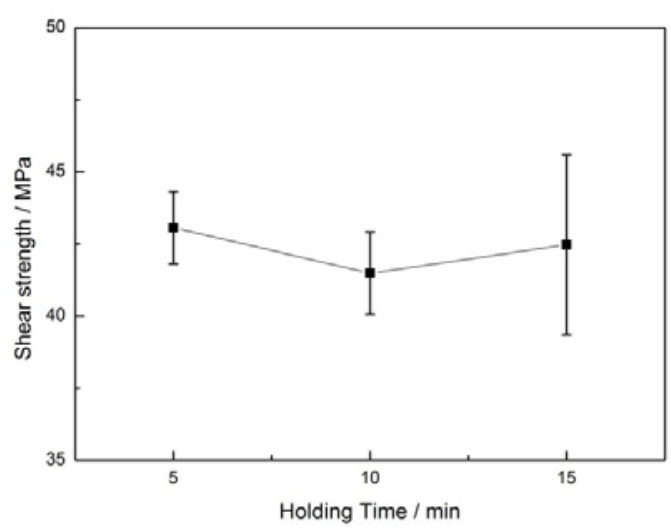

(b) Different holding time

Fig.4 Bonding strength (shear strength) of the interface changed with different conditions

The sintering temperature and holding time impact on the bonding strength of interface is investigated and shown in Fig.4. It can be seen that the interface shear strength increases with the sintering temperature increase in Fig.4 (a). The explanation is: because of the press procedure before sintering, less cracks or gaps will be formed in the interface. With the sintering temperature increase, the copper inter-diffusion is more active. It results in the increase of interface bonding strength. Fig.4 (b) shows that the interface shear strength doesn't change apparently or regularly with holding time increase. It is a minor difference to the traditional point of view. The possible reason is the holding time in this experiment is too short to reveal the difference.

Further, microwave seems to devote a significant contribution to the high interface bonding strength. Due to the selective heating and the dense surface reflection characteristic of microwave, most heating energy is concentrated in the interface. It may also induce a local melt in some place of the interface. This will help to increase the bonding strength. The detail mechanism needs a further research in the future.

\section{Conclusion}

Base on the microwave sintering technology, CuW alloy is successfully coated on copper substrate. Morphology observation and wedged load test show that the CuW coating bonds firmly to the copper substrate without any gap or crack. The interface bonding strength increases with the temperature increase, but doesn't show an obvious regularity changing with holding time increase. 


\section{Acknowledgement}

In this paper, the research is supported by the Natural Science Foundation of Fujian Province Youth Innovation (2012J05093), Specialized Research Fund for the Doctoral Program of Higher Education (20133501120001), the Fundamental Research Funds for the Central Universities (JB-ZR1210), and Project of Fujian Science and Technology Innovation Platform (2011H2003).

\section{References}

[1] Jiten Das, A.Chakraborty, T.P.Bagchi, Bijoy Sarma. Improvement of machinability of tungsten by copper infiltration technique [J]. International Journal of Refractory Metals and Hard Materials. 2008, 26(6): 530-539

[2] Yingli Guo, Jianhong Yi, Shudong Luo, Chengshang Zhou, Lifang Chen, Yuandong Peng. Fabrication of W-Cu composites by microwave infiltration [J]. Journal of Alloys and Compounds. 2010, 492:L75-L78

[3] Pingan Chen, Guoqiang Luo, Qiang Shen, Meijuan Li, Lianmeng Zhang. Thermal and electrical properties of W-Cu composite produced by activated sintering [J]. Materials \& Design. 2013, 46: 101-105

[4] Gavin Whittaker, Michael Mingos. Microwave-assisted solid-state reactions involving metal powders [J] . Journal of Chemistry Society, 1995, 12: 2073-2079

[5] Rustum Roy, Dinesh Agrawal, Jiping Cheng, Shalva Gedevanishvili. Full sintering of powdered-metal bodies in a microwave field [J] . Nature, 1999, 399:668-670

[6] Xiaowei Wang, Kaiyong Jiang, Jiliang Zhang, Fei Wang, Jingjing Zhang. An experimental study on microwave welding technology of $\mathrm{CuW}$ to $\mathrm{Cu}$ Substrate [J]. Advanced Materials Research. 2013, 703:79-85

[7] Qifang Zhu, Xiumin Chang, Beiling Shao, Ansheng Liu, Fusheng Wang, Jinbo Zhao, Lihong Sun, Wei Yao. Evaluation for adhesion strength of hard coatings by wedged load test method [J]. Physical Testing and Chemical Analysis : Part A (Physical Testing), 2000, 36(11): 497-500 